Editor Andreia Biolo, MD

Hospital de Clínicas de Porto Alegre, Internal Medicine/Cardiology Section,

Rua Ramiro Barcelos 2350 Porto Alegre/RS 90035-003, Brazil.

E-mail: abiolo@hcpa.ufrgs.br

\title{
The American Society of Hypertension
}

http://www.ash-us.org

The American Society of Hypertension (ASH) is the largest US organization dedicated exclusively to hypertension and related cardiovascular disease. ASH's mission is to promote strategies to prevent hypertension and to improve the care of patients with hypertension and associated disorders. In addition to information about the Society, the website provides links to the PDF version of several guidelines on hypertension and associated conditions, continuing medical education activities, scientific meetings, and ASH publications (Journal of the American Society of Hypertension and Journal of Clinical Hypertension).

\section{European Society of Hypertension}

http://www.eshonline.org/index.php

The website for the European Society of Hypertension (ESH) has four sections, with information about the ESH, the annual meeting sponsored by the society, and education sections for health professionals and for patients. In the section on ESH Education, one can find hypertension-related guidelines, teaching seminars, links to hypertension-related journals, and highlights from recent publications on hypertension (some downloads require free registration).

\section{Agency for Healthcare Research and Quality: Evidence-Based Practice} http://www.ahrq.gov/clinic/epcix.htm

The establishment of evidence-based practice centers (EPCs) is an initiative of the Agency for Healthcare Research and Quality to promote evidence-based practice in everyday care. The EPCs develop evidence reports and technology assessments on several relevant topics, trying to synthesize the evidence and facilitate the translation of evidence-based research findings. The centers apply systematic review methodology, including meta-analysis. In the website, information about the program, participating centers, and topic nomination and selection are available, along with free downloads of all completed reports. There is a section for Heart and Vascular Disease, including hypertension, coronary artery disease, heart failure, and several other interesting topics. The reports evaluate several questions related to each topic. For example, the brain natriuretic peptide (BNP) and N-terminal pro-BNP report includes the evaluation of their determinants, their diagnostic performance in heart failure patients, their predictive ability with respect to mortality and other cardiac end points, and their value in monitoring heart failure treatment.

\section{World Health Organization: The Atlas of Heart Disease and Stroke}

http://www.who.int/cardiovascular_diseases/resources/atlas

The World Health Organization, in collaboration with the US Centers for Disease Control and Prevention, produced the Atlas of Heart Disease and Stroke as part of an effort to provide actionable information for developing and implementing appropriate policies to reduce the burden of heart disease and stroke. The atlas addresses the global epidemic of heart disease and stroke and provides information about cardiovascular disease and risk factors, the health and economic burden related to it, and organizations and initiatives around the world. Also, it has a "Future and the past" section, with the milestones in knowledge of heart and vascular disorders and estimates for the future of cardiovascular disease. It is easy to download the PDF version, with visual and colorful information on any of those topics. 